\title{
ONE-ONE POLYNOMIAL MAPS
}

\author{
DONALD J. NEWMAN
}

It is a fundamental fact about simultaneous linear equations that uniqueness implies existence in the sense that if the $n \times n$ system $M X=Y$ has, for each $Y$, at most one solution $X$, then, for each $Y$, the equation actually has a solution. Our purpose is to show that this situation persists for certain "nonlinear equations" as well, namely that, restricting everything to the reals, if $P_{1}, P_{2}$ are polynomials and for each $y_{1}, y_{2}$ the system

$$
\begin{aligned}
& P_{1}\left(x_{1}, x_{2}\right)=y_{1}, \\
& P_{2}\left(x_{1}, x_{2}\right)=y_{2},
\end{aligned}
$$

has at most one solution $x_{1}, x_{2}$, then the system always does have a solution.

Thus, we prove in detail the case $n=2$; the general case may follow similarly, but there are some topological difficulties which we have been unable to overcome. Our result is, then,

Theorem. Let $P(x, y)$ and $Q(x, y)$ be polynomials. If the mapping of the plane into the plane given by $(x, y) \rightarrow(P(x, y), Q(x, y))$ is 1-1 then it is onto.

Lemma 1 (Elimination theORy). The number of solutions in the complex plane, counting multiplicities, of $P(x, y)=0, Q(x, y)=0$ is equal to the degree of the resultant $R(x)$, providing

(1) $P$ and $Q$ do not have a common factor, and

(2) Neither the leading form of $P$ nor of $Q$ has $x$ as a factor.

For a complete discussion of the concepts involved in this lemma see [1].

For later convenience, we introduce the

Definition. If a proposition $p(a, b)$ depending on the ordered couple $(a, b)$ is true except for finitely many couples, then we shall say that $p$ holds USUALLY.

Lemma 2. Suppose $P$ and $Q$ are algebraically independent, ${ }^{1}$ then $P-a$ and $Q-b$ have no factor in common USUALLY.

Received by the editors January 23, 1960. $\phi \equiv 0$.

${ }^{1} P$ and $Q$ are algebraically independent if $\phi(P, Q) \equiv 0, \phi$ a polynomial implies 
Proof. Let the maximum of the degrees of $P$ and $Q$ be $n$. We may clearly assume $n>1$. We shall show, in fact, that the exceptional set, $S$, has less than $(n-1)(2 n+1)$ couples, otherwise, choose $(n-1)(2 n+1)$ couples $(a, b)$ from $S$ and let $\phi(u, v)$ be a nontrivial polynomial of $2(n-1)$ th degree which vanishes at all those couples. [That $\phi$ exists follows from the fact that the condition that $\phi$ vanish at all the $(a, b)$ gives $(n-1)(2 n+1)$ homogeneous linear equations in the coefficients, their number being namely $((2 n-1)(2 n)) / 2$ $=(n-1)(2 n+1)+1$.] Now form $\phi(P, Q)$, this has degree $\leqq 2(n-1) \cdot n$. Calling $f_{a, b}$ the common factor of $P-a$ and $Q-b$ for $(a, b)$ one of our chosen couples, we note that $f_{a, b}$ divides $\phi(P, Q)-\phi(a, b)=\phi(P, Q)$. Furthermore, all the $f_{a, b}$ are relatively prime since if $(a, b)$ $\neq\left(a^{\prime}, b^{\prime}\right)\left(a \neq a^{\prime}\right.$, say) then $M f_{a, b}=P-a, M^{\prime} f_{a^{\prime}, b^{\prime}}=P-a^{\prime}$ and we have $\left(M /\left(a^{\prime}-a\right)\right) f_{a, b}-\left(M^{\prime} /\left(a^{\prime}-a\right)\right) f_{a^{\prime}, b^{\prime}}=1$. Hence $\phi(P, Q)$ is divisible by $\prod_{(a, b)} f_{a, b}$ which has degree $\geqq(n-1)(2 n+1)>2(n-1) \cdot n \geqq$ degree of $\phi(P, Q)$. It follows that $\phi(P, Q)$ is identically 0 and the lemma is proved by this contradiction.

Lemma 3. Let $R(x, y, z)$ be a polynomial of $x$ degree $N$ such that USUALLY $R(x, a, b) \not \equiv 0$. There exists a polynomial $A(y, z)$ such that $U S U A L L Y \operatorname{deg} R(x, a, b) \equiv N(\bmod 2)$ if $A(a, b) \neq 0, \operatorname{deg} R(x, a, b)$ $\not \equiv N(\bmod 2)$ if $A(a, b)=0$.

Proof. Call $R(x, y, z)=A_{0}(y, z) x^{N}+A_{1}(y, z) x^{N-1}+\cdots$, and let $A_{0}$ have the distinct irreducible factors $P_{1}, P_{2}, \cdots, P_{k}$. For each $i=1,2, \cdots, k$ let $j_{i}$ denote the smallest integer $j$ for which $P_{i}$ does not divide $A_{j}$. (Since $R(x, a, b) \not \equiv 0$ Usually, this $j$ exists and $0<j \leqq N$.) Now define $A=\prod_{i \ni j_{i} \text { odd }} P_{i}$ (the empty product, as usual, being taken equal to 1$)$. If $A_{0}(a, b) \neq 0$, the lemma is trivial, so assume $A_{0}(a, b)=0$. Among those $i$ for which $P_{i}(a, b)=0$, choose one with a maximal $j_{i}$, denoted by $j$. It follows that $A_{0}(a, b)=A_{1}(a, b)$ $=\cdots=A_{j-1}(a, b)=0$, and that Usually $A_{j}(a, b) \neq 0$ (since $P_{i}$ and $A_{j}$ have no factors in common and so have only a finite number of common zeros), hence $\operatorname{deg} R=N-j$. But $j$ is even if $A(a, b) \neq 0$ while $i$ is USUALLY odd if $A(a, b)=0$ and this proves the lemma.

Lemma 4. Suppose $(x, y) \rightarrow(P, Q)$ maps the real plane 1-1 into the real plane, then $P(x, y)=a, Q(x, y)=b$ cannot have a real solution $(x, y)$ of even multiplicity.

Proof. We require certain properties of the multiplicity $m$, of a solution $(x, y)$. So let $P-a=p_{1}^{e_{1}} \cdots p_{k}^{e_{k}}, Q-b=q_{1}^{f_{1}} \cdots q_{r}^{s_{r}}$ be the decompositions into real irreducible factors, $p_{i}, q_{j}$, all distinct, and call $m_{i j}$ the multiplicity of the solution $(x, y)$ for $p_{i}=0, q_{j}=0\left(m_{i j}=0\right.$ 
means $x, y$ is not a solution). Let us first point out that the point $(x, y)$ is not a multiple point on any $p_{i}=0, q_{j}=0$ since by hypothesis neither curve $P=a$ or $Q=b$ can contain a closed curve. We have, therefore (again see [1]),

(1) $m=\sum e_{i} f_{j} m_{i j}$.

(2) $m_{i j}$ even is equivalent to the fact that the real loci $p_{i}=0$, $q_{j}=0$ do not cross each other at $(x, y)$.

Note then that this means that $m_{i j}$ is even if either of the real loci $p_{i}=0$ or $q_{j}=0$ do not contain $(x, y)$ or contain merely an isolated point at $(x, y)$. We say in this case that the situation is degenerate.

Next, we will show that there is precisely one $i$ and $j$ for which the situation is nondegenerate. We will then show that, for this $i$ and $j, e_{i}$ is odd, $f_{j}$ is odd and the curves $p_{i}=0$ and $q_{j}=0$ do cross one another. This, of course, will suffice to prove the lemma, by (1) and (2) above.

I. There is at least one such $i, j$.

If not then either $P-a$ or $Q-b$ has an isolated zero at $(x, y)$, say $P-a$ does, hence the map, by $P$, of some open disc about $(x, y)$ is not an open interval. But by assumption $(x, y) \rightarrow(P, Q)$ is continuous (1-1), and hence open. This is a contradiction.

II. There is at most one such $i, j$.

Suppose not, then, without loss of generality, we may assume that $P=a$ has two real "branches" through $(x, y)$. As before, the map being open, we can find on each of these branches a point where $Q=b+\epsilon$. This, however, gives two points mapping into $(a, b+\epsilon)$.

III. For this $(i, j)$ both $e_{i}$ and $f_{j}$ are odd.

Suppose e.g., that $e_{i}$ is even, it follows that throughout a neighborhood of $(x, y), P-a$ does not change sign. This again contradicts the openness of the map.

IV. The real loci $p_{i}=0$ and $q_{j}=0$ do cross each other.

Take a small open disc $\Delta$ about $(x, y)$. It suffices to show that in $\Delta$ there exist points where $P>a, Q>b ; P<a, Q>b ; P>a, Q<b$; $P<a, Q<b$. Suppose not, e.g., that $P>a, Q>b$ never occurs, then $(P-a)(Q-b) \leqq 0$ throughout $\Delta$ and this contradicts the fact that $(x, y) \rightarrow(P, Q)$ and $(P, Q) \rightarrow(P-a)(Q-b)$ are both open maps.

By our previous remarks, the proof is complete.

Proof of Theorem. We may arrange matters, by rotation in the $(x, y)$ plane, so that the leading forms of $P$ and $Q$ are neither of them divisible by $x$. From the hypothesis it follows that $P$ and $Q$ are algebraically independent, and so, by Lemma 2, it follows that Lemma 1 is USUALLY applicable to the equations $P-a=0, Q-b=0$. Let the 
resultant be $R(x, a, b)=A_{0} x^{N}+A_{1} x^{N-1}+\cdots, A_{i}=A_{i}(a, b)$ polynomials, $A_{0} \not \equiv 0$.

We first show that $N$ is odd. Since the mapping is 1-1 there exists a (real) point $\left(x_{0}, y_{0}\right)$ for which

(1) $\partial(P, Q) / \partial(x, y) \neq 0$,

(2) $A_{0}\left(P\left(x_{0}, y_{0}\right), Q\left(x_{0}, y_{0}\right)\right) \neq 0$,

(3) $\left(P\left(x_{0}, y_{0}\right), Q\left(x_{0}, y_{0}\right)\right) \in S$, ( $S$ defined as in Lemma 2$)$.

By (1) and the hypothesis, we see that $P=P\left(x_{0}, y_{0}\right), Q=Q\left(x_{0}, y_{0}\right)$ has precisely one real solution of multiplicity 1 , the nonreal solutions come in conjugate pairs in that if $x, y$ is a solution then $\bar{x}, \bar{y}$ is a solution with the same multiplicity. Thus, by Lemma $1, R\left(x, P\left(x_{0}, y_{0}\right)\right.$, $\left.Q\left(x_{0}, y_{0}\right)\right)$ has odd degree. By (2), however, this degree is $N$ and so $N$ is odd.

We can use Lemma 3 (since by Lemma 2 we know that $R(x, a, b)$ is USUALLY not identically 0$)$ and we obtain an $A(a, b)$ such that USUALLY

$P=a, Q=b$ has an odd number of complex solutions for $A(a, b) \neq 0$, $P=a, Q=b$ has an even number of complex solutions for $A(a, b)=0$.

From our remarks about complex conjugates, together with Lemma 4 , we conclude that the image of the real plane under $(x, y) \rightarrow(P, Q)$ consists of the plane less a finite set minus an algebraic curve $(A(P, Q)=0)$ less a finite set. If we finally take into account the topological fact that a 1-1 continuous map of the plane into itself is a homeomorphism, we see easily that the finite sets and the plane curve must be empty since their existence would contradict respectively simple connectedness, openness, and connectedness. We conclude that the image is the whole plane and the proof is complete.

It would be interesting to obtain a purely algebraic proof of this theorem, namely this might extend the result to the case of $(x, y)$ $\rightarrow(P, Q)$ where $x$ and $y$ lie in a field which has an algebraically complete, algebraic extension. Our methods certainly cannot hope to handle this more general situation.

We wish to thank L. Greenberg for his assistance and stimulating discussions.

\section{REFERENCE}

1. R. J. Walker, Algebraic curves, Princeton Mathematical Series vol. 13, Princeton, 1950 , p. 140 et seq.

BROWN UNIVERSITY AND

aVCO, Wilmington, Massachusetts 